\title{
Plasma assisted nitriding for micro-texturing onto martensitic stainless steels*
}

\author{
Takahisa Katoh ${ }^{1}$, Tatsuhiko Aizawa ${ }^{2, *}$, and Tetsuya Yamaguchi ${ }^{3}$ \\ 1 Graduate School of Engineering, Shibaura Institute of Technology, 3-9-14 Shibaura, Minato-City, Tokyo 108-8548, Japan \\ 2 Department of Engineering and Design, Shibaura Institute of Technology, 3-9-14 Shibaura, Minato-City, Tokyo 108-8548, Japan \\ 3 R \& D Center, Sanko-Light Industries, Co. Ltd., 6-22-10 Uekotanaka, Nakahara-City, Kawasaki 211-0053, Japan
}

Received 2 January 2015 / Accepted 16 February 2015

\begin{abstract}
Micro-texturing method has grown up to be one of the most promising procedures to form micro-lines, micro-dots and micro-grooves onto the mold-die materials and to duplicate these micro-patterns onto metallic or polymer sheets via stamping or injection molding. This related application requires for large-area, fine micro-texturing onto the martensitic stainless steel mold-die materials. A new method other than laser-machining, micro-milling or micro-EDM is awaited for further advancement of this micro-texturing. In the present paper, a new micro-texturing method is developed on the basis of the plasma assisted nitriding to transform the two-dimensionally designed micropatterns to the three dimensional micro-textures in the martensitic stainless steels. First, original patterns are printed onto the surface of stainless steel molds by using the dispenser or the ink-jet printer. Then, the masked mold is subjected to high density plasma nitriding; the un-masked surfaces are nitrided to have higher hardness, $1400 \mathrm{Hv}$ than the matrix hardness, $200 \mathrm{Hv}$ of stainless steels. This nitrided mold is further treated by sand-blasting to selectively remove the soft, masked surfaces. Finally, the micro-patterned martensitic stainless steel mold is fabricated as a tool to duplicate these micro-patterns onto the plastic materials by the injection molding.
\end{abstract}

Key words: Micro-texturing, Plasma nitriding, Martensitic stainless steel, Mask-printing, Sand-blasting, Mold-die for injection molding

\section{Introduction}

Micro-machining and micro-EDM (Electric DischargeMachining) have been utilized to form a concave micro-pattern on metals for reduction of the friction coefficient on the tool surface [1]. Short-pulse laser machining has become a reliable means to make fine micro-patterning onto metals and ceramics [2]. The LIGA (Lithographie Galvanoformung Abformung)process as well as the photo-lithography processes turn to be a standard way to make fine sub-micro and nano-texturing onto the die substrate for micro-patterning and nano-patterning [3]. Furthermore, an oxygen plasma etching is utilized for this micro- and nano-texturing onto carbon-based materials such as DLC (Diamond-Like Carbon), CNT (Carbon Nano-Tube) or diamond coatings [4-6]. As the common feature to those

\footnotetext{
* Implications and influences: The present paper has contribution to provide a new manufacturing method for micro-texturing of mold-dies with much reduction of leading time. This micro-textured molds are directly used for injection molding. Micro-patterned plastic cover cases for mobile phones are fabricated.

*e-mail: taizawa@sic.shibaura-it.ac.jp
}

processes, a metallic or a ceramic substrate material is finely machined or etched to leave the negative micro- and nanotextures of original pattern onto these materials. Furthermore, in recent, a new idea was proposed to make positive microtexturing onto steels with use of lattice expansion in their micro-structure via the plasma assisted nitriding [7]. However, this idea was still limited to experiments in trial since the nitriding front end advanced even below the masked regions; the anisotropic diffusion of nitrogen atom solutes might be necessary to realize this positive micro-texturing as had been pointed out in [8]. Owing to an extraordinary nitrogen content as a solute in austenitic stainless steel, the unmasked region of nitrided steel surface turns to be a convex pattern.

The authors have been developing a high density plasma nitriding system to make low-temperature nitriding for various kinds of steels and metals [9-12]. Different from the conventional DC/DC-pulsed or RF plasma nitriding processes, the electron and nitrogen ion densities can be much increased to the order of $10^{16}-10^{17} \mathrm{~m}^{-3}$ uniformly in the chamber. This high density nitrogen plasma state enables us to make efficient nitriding of tool steels, stainless steels, aluminium and titanium alloys [8]. 
In the present paper, this high density plasma nitriding is applied to negative micro-texturing into the martensitic stainless steel mold-substrate. In this new method, a designed or tailored micro-pattern is directly printed onto the polished surface of mold-substrate. This printed micro-pattern is utilized as a mask to prevent from nitriding; the un-masked mold-substrate surface is selectively nitrided and hardened by the nitrogen solid solution in the martensitic stainless steel mold.

First in this paper, the experimental procedure of plasmanitriding assisted micro-texturing is explained with comments on the inner nitriding behavior. To be discussed in later, the holding temperature must be below $723 \mathrm{~K}$ (or $450{ }^{\circ} \mathrm{C}$ ) to be free from the precipitation reaction of chromium in the stainless steels and the formation of chromium nitrides $(\mathrm{CrN})$. The nitriding plasma state is quantitatively described by the plasma diagnosis. The plasma nitriding behavior for AISI-SUS420 is investigated by variation of hardness depth profile with the nitriding time. The mask-taped AISI-SUS420 specimen is employed to demonstrate that the unmasked regions on the specimen surface are selectively hardened by plasma nitriding. The micro-dot patterns are printed on the original AISI-SUS420 specimen surface by using the dispenser. Through the low temperature nitriding, these printed micro-patterns are free from nitriding or hardening; the whole specimen other than micro-dot patterns are nitrided and hardened. The original two dimensional micro-dot patterns transform to a three dimensional micro-dimple texture into the martensitic stainless steel molds by selectively digging them via the sand-blasting.

\section{Experimental procedure}

The plasma assisted nitriding system is mainly explained in this section together with comments on the observation, measurement, test-pieces and the mold-die specimen. In particular, the sand-blasting system is also stated with explanation on the blasting conditions.

\subsection{Plasma assisted nitriding system}

Different from the DC- or RF-plasma generators, where the plasmas are ignited and generated in the frequency of 13.56 MHz or its multiples, the present high density nitriding system has no mechanical matching box with slow response time of $1 \mathrm{~s}$ to $10 \mathrm{~s}$ to adjust the applied power. Since both the input and output powers are automatically matched by frequency adjustment around $2 \mathrm{MHz}$, the matching response time is only limited to $1 \mathrm{~ms}$ at most. This prompt power control provides to make full use of mesoscopic plasma pressure range over $50 \mathrm{~Pa}$. Different from the conventional processes, the vacuum chamber is electrically neutral so that RF-power and DC-bias should be controlled independently from each other. A dipole electrode is utilized to generate RF-plasma; DC bias is directly applied to the specimens. Heating unit is located under this DC-biased cathode plate.

In the following nitriding experiments, the specimens are located on the cathode table before evacuation down to the base pressure of $0.1 \mathrm{~Pa}$. Then, nitrogen gas is first introduced as a carrier gas for heating. After heating to the specified holding
Table 1. A standard high density RF-DC plasma nitriding condition.

\begin{tabular}{ll}
\hline Process & \multicolumn{1}{c}{ Parameters } \\
\hline Pre-sputtering & DC $(500 \mathrm{~V})$ \\
& Nitrogen $(75 \mathrm{~Pa})$ at $693 \mathrm{~K}$ for $1.8 \mathrm{ks}$ \\
Nitriding & RF $(250 \mathrm{~V}), \mathrm{DC}(300 \mathrm{~V})$ \\
& Nitrogen $(100 \mathrm{~mL} / \mathrm{min})$ \\
& Hydrogen $(20 \mathrm{~mL} / \mathrm{min})$ \\
& $75 \mathrm{~Pa}$ at $693 \mathrm{~K}$ for $14.4 \mathrm{ks}$ \\
\hline
\end{tabular}

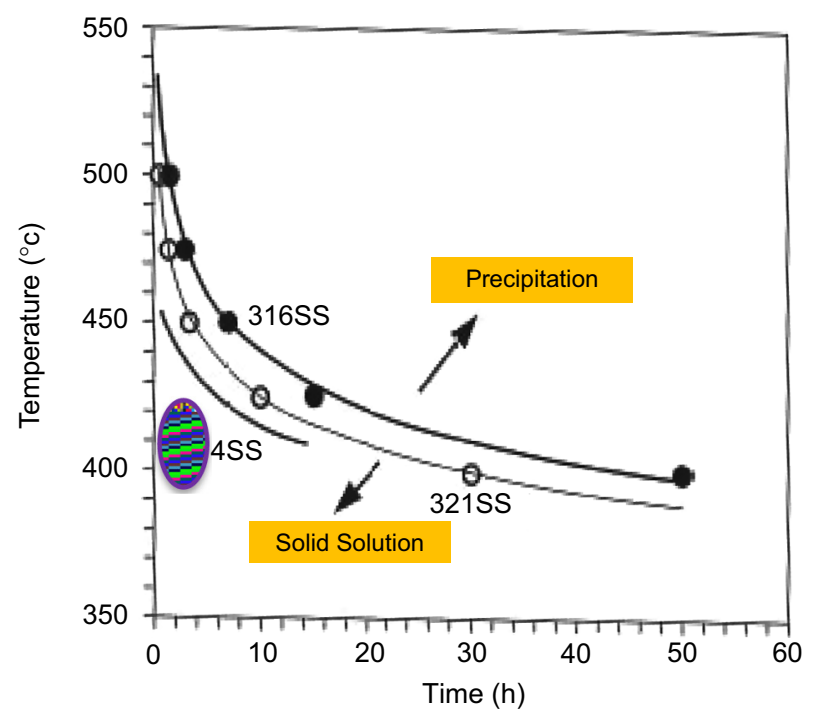

Figure 1. Effect of the holding temperature and the nitriding duration time on the inner nitriding behavior of the austenitic stainless steels.

temperature, the nitrogen pre-sputtering is started at the constant pressure. After pre-sputtering, the hydrogen gas is added to nitrogen gas with the specified partial pressure ratio. Both pressure $(P)$ and temperature $(T)$ controls are automatically performed with the tolerance of $\Delta P<1 \mathrm{~Pa}$ in deviation of partial pressure and $\Delta T<1 \mathrm{~K}$ in temperature fluctuation.

Table 1 summarizes the experimental conditions for present plasma nitriding. In particular, the partial pressure ratio of nitrogen to hydrogen is constant by five to one as a standard condition by controlling the gas flow rate to be $100 \mathrm{~mL} / \mathrm{min}$ for $\mathrm{N}_{2}$ gas and $20 \mathrm{~mL} / \mathrm{min}$ for $\mathrm{H}_{2}$ gas, respectively. The holding temperature is $693 \mathrm{~K}$ or $420{ }^{\circ} \mathrm{C}$.

In reference [13], the effect of the holding temperature and the nitriding duration time on the inner nitriding process was discussed for the austenitic stainless steels. Figure 1 summarizes the whole data on the nitrided austenitic stainless steels. For an example, in case of the austenitic stainless steel type $316 \mathrm{~L}$ by the plasma nitriding for $21.6 \mathrm{ks}$ or $6 \mathrm{~h}$, less precipitation reactions to $\mathrm{CrN}$ take place when the holding temperature $\left(T_{\mathrm{H}}\right)$ becomes below $723 \mathrm{~K}$ or $450{ }^{\circ} \mathrm{C}$. On the other hand, the austenitic stainless steels are nitrided and hardened by the precipitation hardening process when $T_{\mathrm{H}}>723 \mathrm{~K}$. Hence, whenever $T_{\mathrm{H}}$ is kept to be sufficiently lower than this critical diagram in Figure 1, the infiltrating nitrogen atoms into the stainless steels are present as a solute in the matrix, which is hardened by the solid-solution hardening process. 
Since the martensitic stainless steels have BCC' structure, the above directions for nitriding of austenitic stainless steels might be not just suitable to the present nitriding process. As pointed out in reference [14], both the chromium nitride $(\mathrm{CrN})$ and the ion nitrides $\left(\mathrm{Fe}_{2} \mathrm{~N}\right.$ and $\left.\mathrm{Fe}_{4} \mathrm{~N}\right)$ were never formed when the holding temperature was kept constant at $693 \mathrm{~K}$ or $420{ }^{\circ} \mathrm{C}$ even in the case of nitriding the martensitic stainless steels.

\subsection{Test-pieces}

Two types of AISI-SUS420 specimens were prepared for description of plasma nitriding behavior and for micro-texturing with use of masking. A square sample with $25 \times 25 \times 5 \mathrm{~mm}^{3}$ as well as a circular disc sample with the diameter of $25 \mathrm{~mm}$ and the thickness of $5 \mathrm{~mm}$, were commonly used to measure the hardness depth profile in the nitrided specimen. A masked sample by the carbon tape was also employed to prove that unmasked part of AISI-SUS420 surface is selectively nitrided by the present method.

\subsection{Mold-die specimen}

Rectangular AISI-SUS420 plate with $70 \times 40 \times 10 \mathrm{~mm}^{3}$ was prepared to demonstrate that the printed mask pattern was preserved even after plasma nitriding at $693 \mathrm{~K}$. UV-sensitive plastic compounds with the primer were used as an ink to print the initial mask-pattern on this specimen.

\subsection{Observation and measurement}

Optical microscope and scanning electron microscope (SEM) were used to observe the change of printed maskpattern before and after nitriding and sand-blasting. The surface profilometer (Keyence, Co. Ltd.) was also employed to make three dimensional profile after sand-blasting. Micro-Vickers hardness testing was performed to describe the hardening process.

\subsection{Sand blasting method}

In the preliminary survey and experiments, the sandblasting system to make gradual machining into the steel specimens was selected together with optimization of the blasting conditions. The air-blasting system with the SFC-100 (Fuji-Seiki, Co. Ltd.) was utilized to make sand-blasting to eliminate the printed patterns and to dig the printed patterns. Among several candidate media, the glass powders with the diameter of $30 \mu \mathrm{m}$ was employed in this blasting treatment. These media were shot onto the surface of specimen by the skewed angle of $30-45^{\circ}$ with the velocity of $3 \mathrm{~m} / \mathrm{s}$.

\section{Experimental results}

\subsection{Plasma nitriding}

The emissive light spectroscopy as well as the Langmuir probe methods provide a strong tool to quantitatively describe

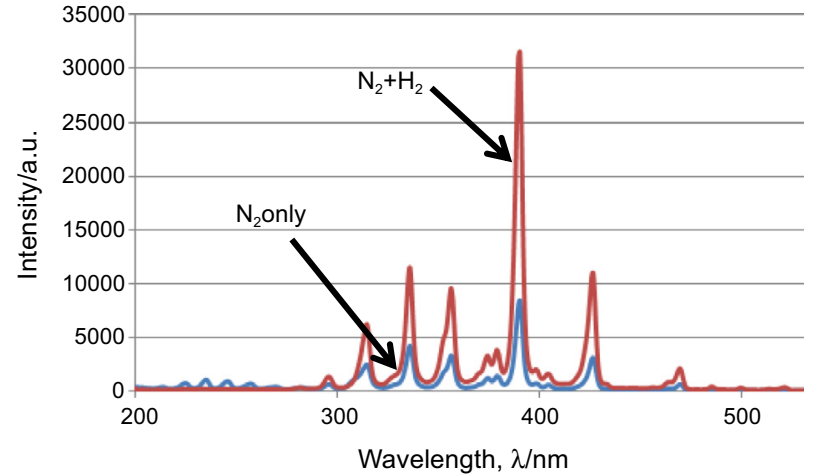

Figure 2. A typical emissive light spectrum of nitrogen plasma for two types of carrier gas supply.

the current plasma state in the plasma nitriding. In particular, the above spectroscopy provides the information of activated species in the plasmas. Furthermore, through the real-time detection of the specified species, the external nitriding conditions can be prescribed to maintain the significant nitrogen atom flux into the substrate surface. Figure 2 shows an emissive light spectrum measured for the current nitrogen plasma with and without hydrogen gases. In particular, with addition of the hydrogen gas, the generated species in plasma are nitrogen atoms and ions together with $\mathrm{NH}$ radicals and nitrogen molecular ions. In particular, the peak intensities for activated nitrogen molecules in the wave length $(\lambda)$ range less than $300 \mathrm{~nm}$ are much reduced; those for nitrogen ions and atoms for $300 \mathrm{~nm}<\lambda<450 \mathrm{~nm}$ are much intensified.

In parallel with the wide-range scanning in the above spectroscopy, the narrow-range scanning was also performed with the wave length resolution of $0.1 \mathrm{~nm}$. NH radicals are detected at $\lambda=336 \mathrm{~nm}$ together with $\mathrm{N}_{2}{ }^{+}$, ionized nitrogen molecule, at $\lambda=337 \mathrm{~nm}$. This significant population of $\mathrm{NH}$ radicals might be responsible for high infiltration of nitrogen atoms into the stainless steels. In the following experiments, the effect of hydrogen gas on the infiltration of nitrogen atoms into the stainless steel is investigated by using the pure nitrogen and the mixed gases as a carrier gas in Table 1 .

\subsection{Micro-texturing}

A carbon-tape masking technique was first utilized to demonstrate that selective lattice expansion and hardening takes place in the un-masked stainless steel substrate. As shown in Figure $3 \mathrm{a}$, the masked regions by the carbon tape, remain the original surface of AISI-SUS420 type-J2 martensitic stainless steel and is free from nitriding. Figure $3 b$ depicts the measured micro-Vickers hardness profile along the scanning line in Figure 3a. The measured hardness is nearly equal to the original matrix hardness of $200 \mathrm{Hv}$ in the masked regions of specimen surface. On the other hand, the average hardness reaches $1400 \mathrm{Hv}$ in the un-masked or the nitrided regions. A sharp change in hardness from $200 \mathrm{Hv}$ up to $1400 \mathrm{Hv}$ or from $1400 \mathrm{Hv}$ down to $200 \mathrm{Hv}$ is detected in Figure 3b across the border between the masked and un-masked regions. That is, the masking pattern changes itself onto the hardness pattern on the stainless steel substrate by this plasma nitriding. 

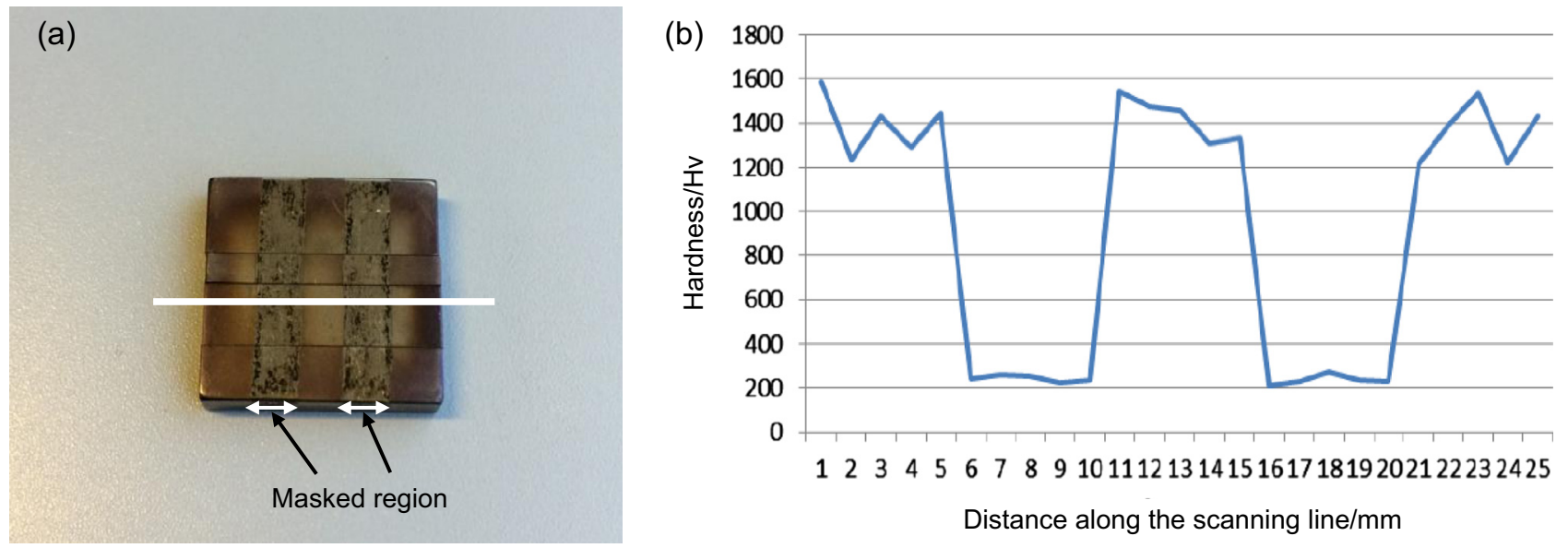

123456678910111213141516171819202122232425

Distance along the scanning line $/ \mathrm{mm}$

Figure 3. Observation and hardness measurement of masked AISI-SUS420 specimen after plasma nitriding for $14.4 \mathrm{ks}$ at $693 \mathrm{~K}$. (a) Plasma-nitrided sample after removal of carbon tapes, and (b) measured hardness profile along the white scanning line in Figure 2 a.

(a)

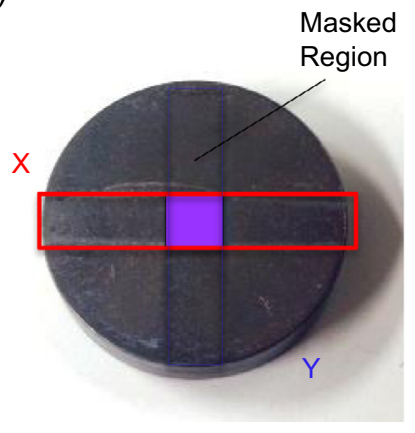

(b)

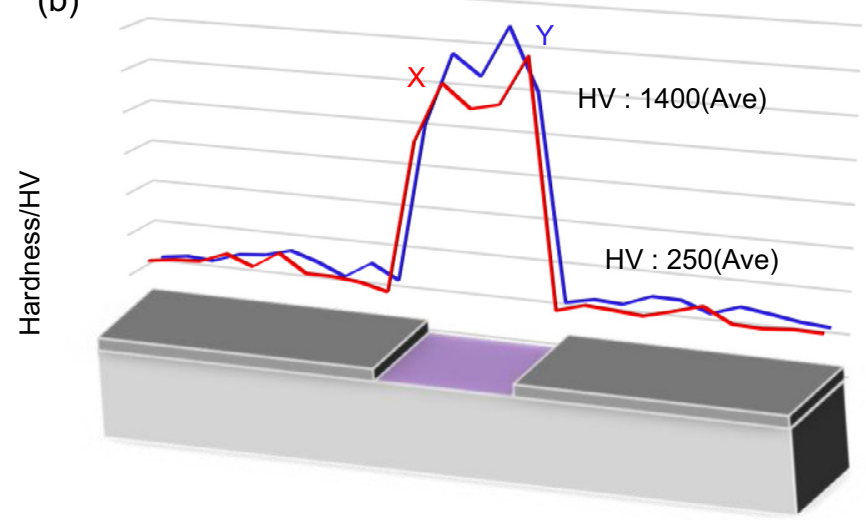

Figure 4. Experimental demonstration on the homogeneous transformation of two dimensional pattern to the hardness profile by the present plasma nitriding. (a) AISI-SUS420 of type J2 with a square mask at the center, and, (b) hardness profiles in the lateral and longitudinal directions across the borders between the masked and unmasked regions.

In the above, the hardness profile was measured along a single scanning line across the borders between the masked and unmasked regions. In order to demonstrate that the original masking pattern should be homogeneously transformed into the hardness profile pattern, a single square region was only left unprinted at the center of AISI-SUS420 type J2 specimen as shown in Figure 4a. That is, the whole surface except for a square region at the center of specimen was printed as a mask. Figure $4 \mathrm{~b}$ compares the hardness profiles measured both in the lateral and longitudinal directions across the mask. Less significant difference was seen in both hardness profiles; i.e. the hardness in the un-masked regions is $1400 \mathrm{Hv}$, and, it remains to be $200 \mathrm{Hv}$ in the masked region.

The steep change of hardness across the edge of masks reveals that the unmasked regions are nitrided to have much higher hardness than matrix hardness of AISI-SUS420 stainless steels. On the other, the masked regions are free from infiltration of nitrogen atoms into matrix. Hence, the hardness map in the nitrided specimen corresponds to the masking pattern.

\subsection{Fabrication of mold-dies}

Micro-dot patterns with the diameter of $100 \mu \mathrm{m}$ as well as the micro-line patterns with the shortest width of $100 \mu \mathrm{m}$, were printed onto the surface of AISI-SUS420 stainless steel molddie unit by using the dispenser. This initially micro-patterned mold-die was subjected to the present high density plasma nitriding by the listed conditions in Table 1. The micro-patterns were used as a mask to prevent from nitriding. Figure 5a depicts the nitrided mold-die specimen. The other surface than the masked micro-dot and micro-line patterns are plasmanitrided to have higher hardness by $1400 \mathrm{Hv}$ than the matrix.

The sand-blasting method was employed to mechanically remove the masked, soft regions. As shown in Figure 5b, the masked regions as well as masking prints are removed from the specimen surface and dug into the depth. This proves that the nitrided surface areas other than the printed micro-patterns remain as they were before blasting. The average machining rate by this blasting process is $10 \mu \mathrm{m} / \mathrm{min}$. 

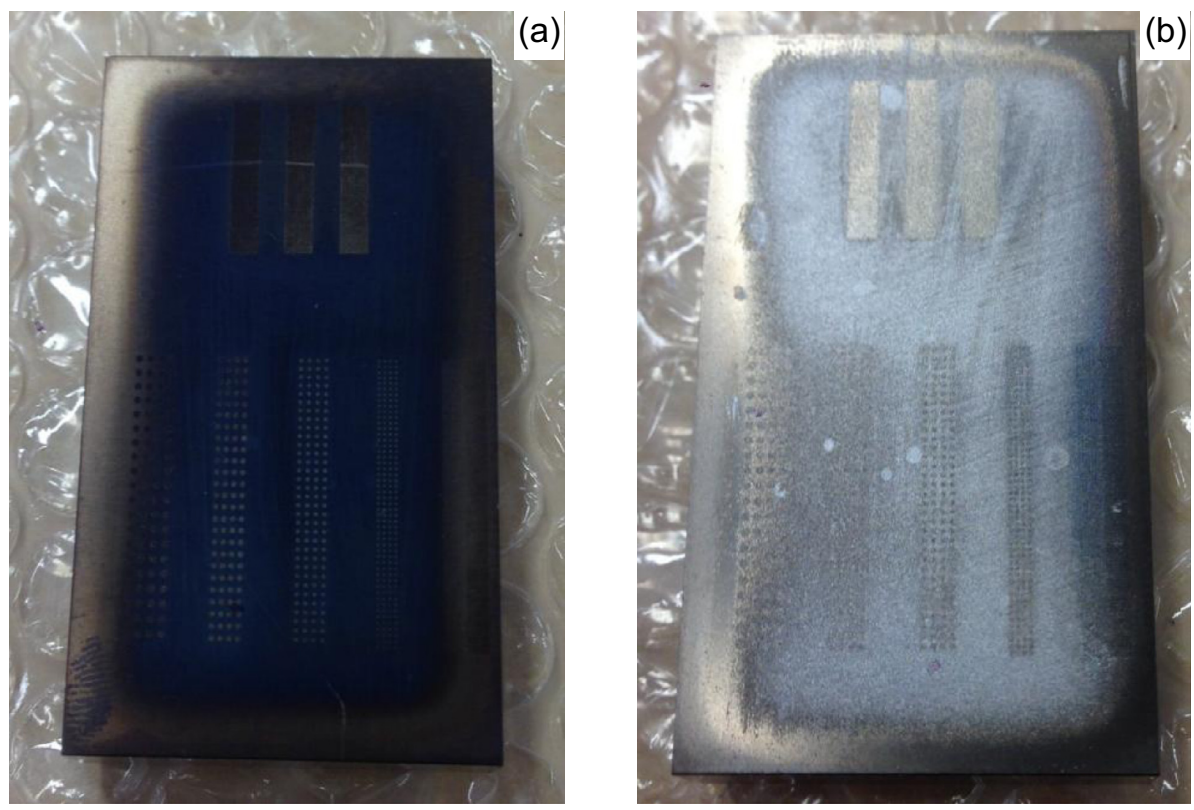

Figure 5. AISI-420 stainless steel mold-die specimen for injection molding. (a) after plasma-nitriding and (b) after sand-blasting.
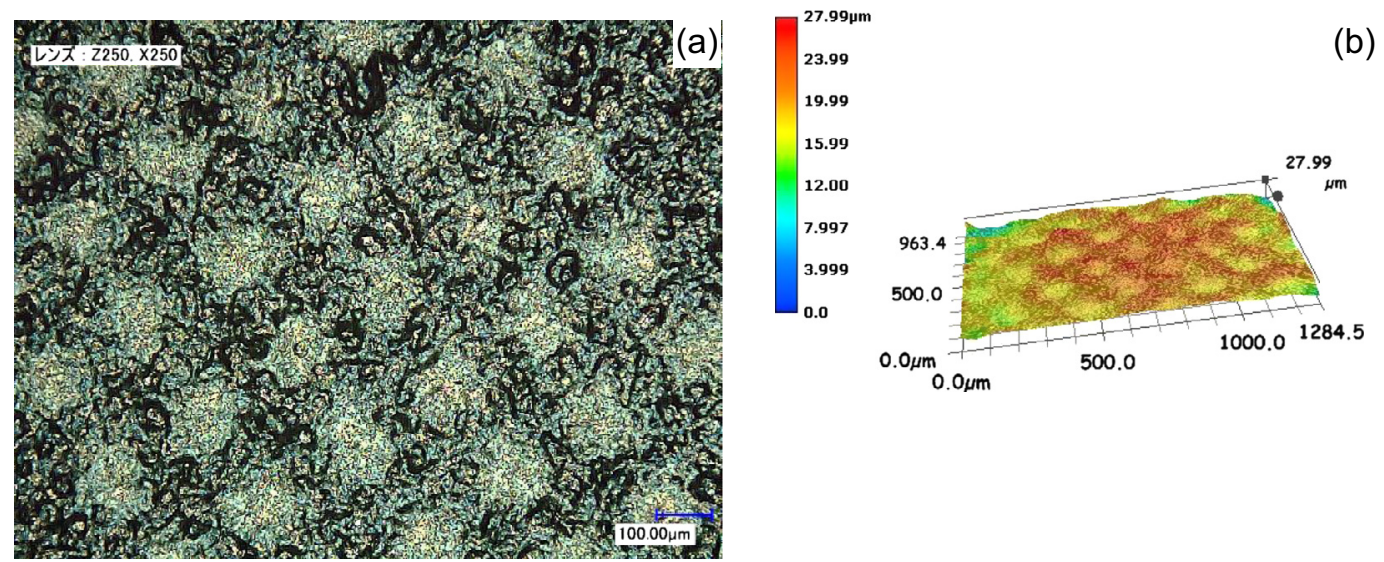

Figure 6. Microscopic image of micro-textured AISI-SUS420 stainless steel mold-die after sand-blasting. (a) Top view of micro-dot pattern, and (b) its surface profile distribution.

Figure 6 depicts its microscopic image and its surface profile distribution on the blasted mold surface, respectively. In correspondence to the micro-dots with the average diameter of $100-110 \mu \mathrm{m}$, the micro-dimples with the same size and dimension are formed on the surface by this sand-blasting. Their average depth reaches to $20-30 \mu \mathrm{m}$. That is, the micro-dimple textures with the same dimension and distribution as printed on the mold-unit surface are formed into the nitrided mold-unit to have accurate depth profile.

\section{Discussion}

Nitrogen atoms infiltrate from the activated species $\left\{\mathrm{N}^{*}, \mathrm{~N}^{+}\right.$and $\left.\mathrm{NH}\right\}$ in the plasmas in Figure 2 to the depth of martensitic stainless steels. After $[8,14]$, these atoms occupy the octahedral vacancy sites in the BCC' crystalline structure of martensitic stainless steels as a nitrogen solute. Owing to extraordinary concentration of nitrogen solutes, the hardness of nitrided regions significantly increases beyond the matrix hardness of $200 \mathrm{Hv}$ in Figure 3. Lattice expansion mainly along the $c$-axis takes place in the BCC'structure with increasing the nitrogen solute concentration. This results in the anisotropic straining of $\mathrm{BCC}^{\prime}$ structure in the $c$-axis; then, the hardness of nitrided layer significantly increases with nitrogen solute concentration during the present plasma nitriding.

In the present method, the un-masked martensitic stainless steel mold is selectively nitrided to have more nitrogen concentration; hence, only the un-masked part of mold is hardened to have much higher hardness, $1400 \mathrm{Hv}$, than the matrix hardness, $200 \mathrm{Hv}$. On the other hand, the masked part of mold remains as it is even during the nitriding process; its hardness is the same 
Table 2. Comparison of the leading time to form the micro-dimple patterns on the stainless steel surface between the end-milling and the present micro-texturing.

\begin{tabular}{lccc}
\hline & Micro end-milling & Time $(\mathrm{ks})$ & Present micro-texturing \\
\hline Preparation of CAD data & $\begin{array}{c}\text { Geometric modeling of 2D } \\
\text { micro-patterns }\end{array}$ & - & $\begin{array}{c}\text { Geometric modeling of 2D } \\
\text { micro-patterns } \\
\text { Building-up of CAM data } \\
\text { from CAD data } \\
\begin{array}{c}\text { Construction of data } \\
\text { for machining }\end{array}\end{array}$ \\
$\begin{array}{l}\text { Fine machining } \\
\text { End-milling process and } \\
\text { polishing }\end{array}$ & 360 & 1000 & $\begin{array}{c}\text { to printing commands } \\
\text { Plasma nitriding and } \\
\text { Sand-blasting }\end{array}$ \\
\hline
\end{tabular}

as the matrix hardness. In other words, the printed micropattern in geometry on the mold surface is transformed to the hardness profile in the mold. That is, just like the development process of negative film to a piece of photo, the designed micro-pattern by using CAD is developed to the three dimensional micro-texture into the stainless steel mold and die for injection molding and stamping.

In the sand-blasting, the capacity to dig the masked area is dependent on the selection of blasting media. In the present study, the silica-glass beads with the average diameter of $30 \mu \mathrm{m}$ were employed and shot to the surface of nitrided stainless steel mold with the shooting velocity of 3-5 m/s. Under this blasting condition, the masked area of mold was selectively removed and dug into the depth without plastic deformation of nitrided surface even at the edge of printed patterns. Hence, as shown in Figures 5 and 6, the micro-dot pattern area, printed on the stainless steel mold, was selectively removed and dug into the micro-dimple textures with less change in geometry on the nitrided area of mold.

Let us estimate the leading time of this micro-texturing with comparison to the conventional micro-milling process. Consider the large area micro-texturing of micro-dimples with the average diameter of $100 \mu \mathrm{m}$, the depth of $30 \mu \mathrm{m}$, and the pitch of $200 \mu \mathrm{m}$, in the similar manner as shown in Figures 5 and 6 . In the latter, the fine end-milling tools with the diameter of $100 \mu \mathrm{m}$ are utilized for direct micro-machining of above micro-dimples onto the stainless steel mold with the area of $100 \times 100 \mathrm{~mm}^{2}$. For simplicity, the leading time to prepare for the CAD data in micro-patterning design is common to both processes. In the present approach, the initial CAD data are automatically transformed into a series of commands for dispenser or ink-jet printer by using the software of "Illustrator". The set-up of dispenser and mold-units as well as inkjet printing are only necessary to print the initial designed micro-patterns onto the mold surface. Total processing time for nitriding is counted to be $14.4 \mathrm{ks}$ including the set-up, the heating and cooling processes. The final sand-blasting requires only for $1.8 \mathrm{ks}$, including the set-up and finishing.

Table 2 compares the leading time for three steps in the large-area micro-texturing between the present method and the micro-milling. In the latter, we assume that the CAM (Computer Aided Machining)-data construction time per a single micro-dimple requires for $0.36 \mathrm{~s}$, including the optimization in the positioning control, and the trial test for data validation. In addition, a single milling process to form a micro-dimple is assumed to be $1 \mathrm{~s}$, also including the positioning control of tools, the set-up and the polishing. Without the time for tool-change and manual operation taken into account, the total leading time by the micro-milling is $1360 \mathrm{ks}$ or $380 \mathrm{~h}$. By using the present micro-texturing, this total leading time is reduced to be $16.2 \mathrm{ks}$ or $1 / 80$ to $1 / 90$ of the above duration by micro-milling. Further to be noticed, the leading time by the present micro-texturing is insensitive, to the area to be machined or the number of micro-patterning units, and, to their geometric complexity.

\section{Conclusion}

High density plasma nitriding assists to make microtexturing onto the martensitic stainless steel mold-die with use of the printed mask-pattern by the dispenser. Two dimensionally designed micro-patterns by CAD are transformed to the three dimensionally machined micro-textures into the stainless steel molds and dies with significantly less leading time than the conventional fine micro-milling. In particular, this microtextured mold-unit is inserted into the cassette mold-die for injection molding process to duplicate the concave micro-textures onto the plastic part surfaces as a convex micro-patterns.

There are many parameters in this micro-texturing method to be optimized toward the accurate development with high resolution in dimension. In particular, the polymer ink has to be formulated to have more heat resistance and to be free from nitrogen atom infiltration through the printed mask on the mold-die material. Through optimization in printing with use of the present dispenser, the diameter of micro-dimple patterns can be reduced to $10-15 \mu \mathrm{m}$ in diameter after nitriding. The plasma-nitriding conditions as well as the selection of blasting media must be also optimized to increase the aspect ratio of depth to diameter in the micro-texture and to control the depth profile in the side of micro-texture units.

Acknowledgements. The authors would like to express their gratitude to Dr. S. Muraishi (TiTech), Mr. K. Modkhua, Ms. C. Yooliengphan, Ms. S. Sukkasem, Mr. T. Aswapanyawongse (KMUTT), and, Ms. B.M. Bouzan, Ms. F.P. Gusmo, Mr. C.V. Albamonte (Brazil Federal Universities) for their help in experiments during their stay at SIT. This study is financially supported in part by METI-project with the contract of $\# 44001$. 


\section{References}

1. I. Etsion, Tribology Letters 17 (2004) 733-737.

2. T. Aizawa, T. Inohara, Proceedings of the 7th International Conference on Micro-Manufacturing 7 (2012) 6-73.

3. Japan Association on Optical-Innovation for Industries, Future vision in optical industries (Advancement and extension in global competition) (2004).

4. T. Aizawa, K. Mizushima, R.T. Retadiono, Y. Sugita, Research Report of Shibaura Institute of Technology 55-2 (2011) 13-22.

5. T. Aizawa, Y. Sugita, Research Report of Shibaura Institute of Technology 56-2 (2012) 47-56.

6. T. Aizawa, T. Fukuda, Surface and Coating Technologies 215 (2013) 364-368.

7. G. Marcos, et al., Surface and Coating Technologies 205 (2011) S275-S279.
8. D.J. Santojojo, et al., Proceedings of the 8th South East Asia Technology University Consortium Conference, Johor Bahru, Malaysia, 2014, CD-ROM.

9. T. Aizawa, et al., Proceedings of the 6th South East Asia Technology University Consortium Conference, Bangkok, 2012, CD-ROM.

10. T. Aizawa, et al., International Steel Research 84 (2012) 739-742.

11. T. Aizawa, et al., Research Report of Shibaura Institute of Technology 57-1 (2013) 1-10.

12. T. Aizawa, et al., Proceedings of the 8th South East Asia Technology University Consortium Conference, Johor Bahru, Malaysia, 2014, CD-ROM.

13. H. Anzai (Ed.), Surface treatment for high qualification of dies and molds, Nikkan-Kougyou Shinbun, 2011.

14. D. Santojoyo, T. Aizawa, S. Muraishi, H. Morita, Proceedings of 9th International Conference on Micro-Manufacturing 90 (2014) 1-8.

Cite this article as: Katoh $\mathrm{T}$, Aizawa $\mathrm{T} \&$ Yamaguchi T: Plasma assisted nitriding for micro-texturing onto martensitic stainless steels. Manufacturing Rev. 2015, 2, 2. 